\title{
Bertrand competition with asymmetric costs: a solution in pure strategies
}

\author{
Thomas Demuynck ${ }^{1} \cdot$ P. Jean-Jacques Herings ${ }^{2} \cdot$ Riccardo D. Saulle $^{2,3}$. \\ Christian Seel ${ }^{2}$
}

Published online: 2 April 2019

(C) The Author(s) 2019

\begin{abstract}
We consider a Bertrand duopoly with homogeneous goods and we allow for asymmetric marginal costs. We derive the Myopic Stable Set in pure strategies as introduced by Demuynck et al. (Econometrica 87:111-138, 2019). In contrast to the set of Nash equilibria, the unique Myopic Stable Set can be easily characterized in closed form and it provides an intuitive set-valued prediction.
\end{abstract}

Keywords Bertrand competition - Asymmetric costs · Myopic stable set

\section{Introduction}

The analysis of price competition is a fundamental part of oligopoly theory since Bertrand's contribution (1883). The Bertrand duopoly with symmetric constant marginal costs, homogeneous goods, and continuous prices has a unique pure strategy Nash Equilibrium characterized by a strategy profile in which prices equal marginal costs.

Thomas Demuynck acknowledges financial support by the Fonds de la Recherche Scientifique-FNRS under Grant no. F.4516.18.

$凶 \quad$ P. Jean-Jacques Herings

p.herings@maastrichtuniversity.nl

Thomas Demuynck

thomas.demuynck@ulb.ac.be

Riccardo D. Saulle

r.saulle@maastrichtuniversity.nl

Christian Seel

c.seel@maastrichtuniversity.nl

1 Ecares, Université Libre de Bruxelles, Brussels, Belgium

2 Department of Economics, Maastricht University, Maastricht, The Netherlands

3 Sapienza University of Rome, Rome, Italy 
If marginal costs are not symmetric across firms and the market is shared if firms set equal prices, no pure strategy Nash equilibrium exists. Blume (2003) shows that there exists a Nash equilibrium in mixed strategies where the more efficient firm sets price equal to the opponent's marginal cost and serves the entire market with probability 1. The rival randomizes on an interval above his marginal cost. Kartik (2011) strengthens the result by showing that all undominated equilibria lead to the same market price and shares. The complete set of undominated Nash equilibria is not constructed in these papers.

In this paper, we analyze the same Bertrand game. We follow Blume (2003) and Kartik (2011) in that we focus on strategy profiles in which no firm chooses a predatory price, i.e., a price below marginal cost. Instead of Nash equilibrium, however, we employ a solution concept recently introduced by Demuynck et al. (2019), the Myopic Stable Set. A set of strategy profiles is myopically stable if it satisfies three conditions, deterrence of external deviations, asymptotic external stability, and minimality. Deterrence of external deviations requires that no player benefits by switching her strategy, such that the resulting strategy profile is outside the Myopic Stable Set. Asymptotic external stability makes sure that, from any strategy profile outside the set, it is possible to get arbitrarily close to a strategy profile inside the Myopic Stable Set by a sequence of better replies. Minimality requires that the Myopic Stable Set is minimal with respect to set inclusion.

In Demuynck et al. (2019), we defined the Myopic Stable Set for a very general class of social environments (Chwe 1994) that allows for infinite state spaces and includes normal-form games as a special case. We proved that if the state space is compact, then the Myopic Stable Set exists, and under some mild continuity assumptions, it is also unique. Moreover, we showed that the Myopic Stable Set coincides with the set of pure strategy Nash Equilibria for supermodular games, aggregative games, and potential games.

In light of these results, the Bertrand model with asymmetric costs is interesting for several reasons: it does not satisfy the compactness and continuity assumptions of Demuynck et al. (2019), it does not belong to any of the aforementioned classes of games, and the set of pure strategy Nash equilibria of this game is empty. Moreover, the set of mixed-strategy Nash equilibria is large.

We prove existence and uniqueness of the Myopic Stable Set for symmetric and asymmetric Bertrand competition. We characterize the set in closed form. The set is small and gives an intuitive prediction.

\section{Model and solution concept}

In this section, we define the Myopic Stable Set for normal-form games $G:=$ $\left(N,\left(S_{i}\right)_{i \in N},\left(\pi_{i}\right)_{i \in N}\right)$, where $N=\{1, \ldots, n\}$ is a finite set of players with typical element $i, S_{i}$ is the set of pure strategies for each player $i \in N$, and $\pi_{i}: \chi_{i \in N} S_{i} \rightarrow \mathbb{R}$ is the payoff function of player $i$. Denote the strategy space by $S=\chi_{i \in N} S_{i} \subseteq \mathbb{R}^{n}$. We use the standard notation $s:=\left(s_{i}, s_{-i}\right) \in S$ to denote the strategy profile where $s_{-i}$ is the list of strategies of all players except $i$, i.e., $s_{-i}:=\left(s_{j}\right)_{j \in N \backslash\{i\}}$. 
We say that a strategy profile $s^{\prime} \in S$ dominates another strategy profile $s \in S$ if there is a player who can unilaterally deviate to $s^{\prime}$ and strictly prefers $s^{\prime}$ over $s$, i.e., from $s$, the player has a better reply, such that the resulting strategy profile is $s^{\prime}$.

Definition 1 (Dominance) Let $s, s^{\prime} \in S$ be two strategy profiles. The strategy profile $s^{\prime}$ dominates $s$ if there exists a player $i \in N$, such that $\pi_{i}\left(s^{\prime}\right)>\pi_{i}(s)$ and $s_{-i}^{\prime}=s_{-i}$.

Let some strategy profile $s \in S$ be given. The set of all strategy profiles that dominates $s$ together with $s$ itself is denoted by $f(s)$ :

$$
f(s)=\{s\} \cup\left\{s^{\prime} \in S \mid s^{\prime} \text { dominates } s\right\} .
$$

We define the twofold composition of $f$ by:

$$
f^{2}(s)=\left\{s^{\prime \prime} \in S \mid \exists s^{\prime} \in S: s^{\prime} \in f(s) \text { and } s^{\prime \prime} \in f\left(s^{\prime}\right)\right\} .
$$

By induction, for $k \geq 3$, we can define the $k$-fold composition $f^{k}(s)$ by $s^{k} \in f^{k}(s)$ if there is $s^{k-1} \in f^{k-1}(s)$, such that $s^{k} \in f\left(s^{k-1}\right)$. Observe that, for all $k, \ell \in \mathbb{N}$, such that $k \leq \ell$, it holds that $f^{k}(s) \subseteq f^{\ell}(s)$. We define the set of all strategy profiles that can be reached from $s$ by a finite number of dominations by $f^{\mathbb{N}}(s)$, where

$$
f^{\mathbb{N}}(s):=\bigcup_{k \in \mathbb{N}} f^{k}(s)
$$

Given $s, s^{\prime} \in S$, we say that a strategy profile $s^{\prime}$ asymptotically dominates $s$ if, starting from $s$, it is possible to get arbitrarily close to $s^{\prime}$ in a finite number of dominations. Let $d\left(s, s^{\prime}\right)$ denote the Euclidean distance between $s$ and $s^{\prime}$. The asymptotic dominance criterion is formally defined as follows:

Definition 2 (Asymptotic dominance) A strategy profile $s^{\infty} \in S$ asymptotically dominates $s \in S$ if, for all $\varepsilon>0$, there exists $s^{\prime} \in f^{\mathbb{N}}(s)$, such that $d\left(s^{\infty}, s^{\prime}\right)<\varepsilon$.

We denote the set of all strategy profiles in $S$ that asymptotically dominate $s$ by $f^{\infty}(s)$. Formally

$$
f^{\infty}(s)=\left\{s^{\infty} \in S \mid \forall \varepsilon>0, \exists s^{\prime} \in f^{\mathbb{N}}(s): d\left(s^{\infty}, s^{\prime}\right)<\varepsilon\right\}
$$

It is easy to see that the set $f^{\infty}(s)$ coincides with the closure of the set $f^{\mathbb{N}}(s)$. We are now ready to define the Myopic Stable Set, abbreviated as MSS, for normal-form games.

Definition 3 (Myopic stable set (MSS)) Let $G:=\left(N,\left(S_{i}\right)_{i \in N},\left(\pi_{i}\right)_{i \in N}\right)$ be a normalform game. The set $M \subseteq S$ is a Myopic Stable Set (MSS) if it is closed and satisfies the following three conditions:

1. Deterrence of external deviations: For all $s \in M, f(s) \subseteq M$.

2. Asymptotic external stability: For all $s \notin M, f^{\infty}(s) \cap M \neq \emptyset$.

3. Minimality: There is no closed set $M^{\prime} \subsetneq M$ that satisfies Conditions 1 and 2 . 
Let $M$ be a MSS. Deterrence of external deviations requires that no player can profitably deviate to a strategy profile outside $M$. Asymptotic external stability requires that any strategy profile outside $M$ is asymptotically dominated by a strategy profile in $M$. Hence, from any strategy profile outside of $M$, it is possible to get arbitrary close to a strategy profile in $M$ by a finite number of dominations. Observe that an empty set would necessarily violate asymptotic external stability, so it follows that $M$ is non-empty.

While we focus on pure strategies, the MSS can be defined analogously on the set of mixed strategies; for details, see Online Supplement A.6 of Demuynck et al. (2019). In any (mixed) Nash equilibrium, we have $f(s)=f^{\infty}(s)=s$. Thus, by asymptotic external stability, all mixed-strategy Nash equilibria are part of the MSS in mixed strategies.

\section{Bertrand duopoly}

Consider a model with two firms, $N=\{1,2\}$, having constant marginal costs $0 \leq$ $c_{1} \leq c_{2}$ and no fixed costs. In many countries, pricing below marginal or average cost is considered to be predatory pricing and is, thus, forbidden by law. Thus, we require the strategy space $P_{i}$ of a firm $i \in N$ to consist of all non-predatory prices, i.e., $P_{i}=\left[c_{i}, \infty\right)$, and we define $P:=P_{1} \times P_{2}$ with typical element $p$.

The absence of predatory pricing is similar to the focus on undominated equilibrium strategies in Kartik (2011). However, pricing at marginal costs is allowed in our setting, while it is ruled out in equilibria considered by Kartik (2011). This difference does not influence the set of undominated Nash equilibria in the asymmetric case $c_{1}<c_{2}$, as, by a standard argument in this literature, no equilibrium in which Firm 2 places mass at $c_{2}$ can exist. However, restricting attention to equilibria with price strictly larger than marginal cost leads to non-existence in the symmetric case which is not particularly appealing.

The continuous market demand function $D:[0, \infty) \longrightarrow \mathbb{R}_{+}$expresses the demand for the good as a function of the lowest price $p_{\mathrm{m}}$ available in the market. There exists a "choke" price $\bar{p}_{\mathrm{m}} \in\left(c_{2}, \infty\right)$, such that $D\left(p_{\mathrm{m}}\right)=0$ for all $p_{\mathrm{m}} \geq \bar{p}_{\mathrm{m}}$. The demand is strictly decreasing on $\left[0, \bar{p}_{\mathrm{m}}\right]$ and zero on $\left[\bar{p}_{\mathrm{m}}, \infty\right)$. We assume that there is an $\varepsilon>0$, such that the profits $\left(p_{\mathrm{m}}-c_{1}\right) D\left(p_{\mathrm{m}}\right)$ of a monopolist with marginal costs $c_{1}$ are strictly increasing in $p_{\mathrm{m}}$ on the domain $\left[c_{1}, c_{2}+\varepsilon\right]$. This assumption ensures an element of competition between the firms. If the profit of a monopolist with marginal cost $c_{1}$ has a maximum for $p_{1} \in\left[c_{1}, c_{2}\right)$, then the strategy profiles in which Firm 1 chooses $p_{1}$ and Firm 2 chooses a strictly higher price are Nash equilibria and part of the MSS.

For each firm $i \in\{1,2\}$, its individual demand depends on its price $p_{i}$ and the price of its competitor $j \neq i$, and is given by:

$$
Q_{i}\left(p_{i}, p_{j}\right)= \begin{cases}D\left(p_{i}\right) & \text { if } p_{i}<p_{j} \\ D\left(p_{i}\right) / 2 & \text { if } p_{i}=p_{j} \\ 0 & \text { if } p_{i}>p_{j}\end{cases}
$$


Thus, the firm setting the lower price serves the entire market. In case of a tie, the market share of each firm equals $1 / 2$. For each firm $i \in\{1,2\}$, the demand $Q_{i}\left(p_{i}, p_{j}\right)$ leads to a payoff function $\pi_{i}: P_{1} \times P_{2} \longrightarrow \mathbb{R}$ defined by $\pi_{i}\left(p_{i}, p_{j}\right)=$ $\left(p_{i}-c_{i}\right) Q_{i}\left(p_{i}, p_{j}\right)$. The normal-form game $\left(\{1,2\},\left(P_{i}\right)_{i \in\{1,2\}},\left(\pi_{i}\right)_{i \in\{1,2\}}\right)$ is denoted by $\Gamma$.

For $c_{1}<c_{2}$, let $\underline{p}_{1} \in\left(c_{1}, c_{2}\right)$ be the price, such that $\pi_{1}\left(\underline{p}_{1}, c_{2}\right)=\pi_{1}\left(c_{2}, c_{2}\right)$. To see that this price is uniquely determined, note that $\pi_{1}\left(c_{1}, c_{2}\right)=0, \pi_{1}\left(c_{2}, c_{2}\right)>0$, and $\pi_{1}\left(p_{1}, c_{2}\right)$ is strictly increasing and continuous for $p_{1} \in\left[c_{1}, c_{2}\right)$ and has a downward jump at $c_{2}$. By the intermediate value theorem, $\underline{p}_{1}$ exists. Since $\pi_{1}\left(p_{1}, c_{2}\right)$ is strictly increasing for $p_{1} \in\left[c_{1}, c_{2}\right)$, we obtain that $\underline{p}_{1}$ is uniquely determined. This allows us to characterize the MSS as follows.

Proposition 4 (i) If $c_{1}=c_{2}=c$, the unique MSS of $\Gamma$ is given by $P^{*}=\left\{p_{1}=\right.$ $\left.p_{2}=c\right\}$.

(ii) If $c_{1}<c_{2}$, the unique MSS of $\Gamma$ is given by

$$
P^{*}=\left\{\left(p_{1}, p_{2}\right) \in P \mid \pi_{1}\left(p_{1}, c_{2}\right) \geq \pi_{1}\left(c_{2}, c_{2}\right), p_{2}=c_{2}\right\}=\left[\underline{p}_{1}, c_{2}\right] \times\left\{c_{2}\right\} .
$$

Proof We first prove Part (i). Deterrence of external deviations is satisfied as $\pi_{1}(c, c)=$ $\pi_{2}(c, c)=0$ and $\pi_{1}\left(p_{1}, c\right) \leq 0, \pi_{2}\left(c, p_{2}\right) \leq 0$. As $f^{\infty}(c, c)=\{(c, c)\}$, the profile $(c, c)$ is part of every MSS. Hence, by minimality, if $P^{*}$ is a MSS, then it is also unique.

It remains to verify asymptotic external stability. Without loss of generality, let $p_{1} \leq p_{2}$. Let some $\left(p_{1}, p_{2}\right) \neq(c, c) \in P$ be given. If $c<p_{1}<p_{2}$, then Firm 2 can profitably deviate to $p_{2}^{\prime}=\left(c+\min \left\{p_{1}, \bar{p}_{\mathrm{m}}\right\}\right) / 2$ and Firm 1 can profitably deviate in the next step to $p_{1}^{\prime}=\left(c+p_{2}^{\prime}\right) / 2$ and so forth. It follows that $(c, c) \in f^{\infty}\left(p_{1}, p_{2}\right) \cap P^{*}$. If $p_{1}=c<p_{2}$, then Firm 1 can profitably deviate to $\left(p_{1}^{\prime}, p_{2}\right)$, where $p_{1}^{\prime} \in\left(c, p_{2}\right)$ is chosen. such that $p_{1}^{\prime}<c+\varepsilon$ with $\varepsilon>0$. such that the profits $\left(p_{\mathrm{m}}-c\right) D\left(p_{\mathrm{m}}\right)$ of a monopolist with marginal costs $c$ are strictly increasing in $p_{\mathrm{m}}$ on the domain $[c, c+\varepsilon]$. We can then continue as in the previous case. If $p_{1}=p_{2}>c$, then there is $p_{1}^{\prime} \in\left(c, p_{2}\right)$, such that $\pi_{1}\left(p_{1}^{\prime}, p_{2}\right)>\pi_{1}\left(p_{1}, p_{2}\right)$. Either $p_{2} \leq \bar{p}_{\mathrm{m}}$ or $p_{1}^{\prime}$ is obtained by slightly undercutting $p_{2}$, or $p_{2}>\bar{p}_{m}$ and any $p_{1}^{\prime} \in\left(c, \bar{p}_{\mathrm{m}}\right)$ will do. Now, we can continue as before. Because of the legal restrictions on predatory pricing, we have covered all strategy profiles in $P \backslash P^{*}$ and thereby shown that $P^{*}$ satisfies asymptotic external stability which completes the proof of (i).

We split the proof of (ii) into several steps. The first step verifies deterrence of external deviations and asymptotic external stability. The remaining steps establish minimality and uniqueness.

Step 1. We show that $P^{*}$ satisfies deterrence of external deviations and asymptotic external stability. We start with deterrence of external deviations. For $\left(p_{1}, c_{2}\right) \in P^{*}$, profits of Firm 1 are greater than or equal to $\pi_{1}\left(c_{2}, c_{2}\right)$. For $p_{1} \notin\left[p_{1}, c_{2}\right]$, profits are $\pi_{1}\left(p_{1}, c_{2}\right)<\pi_{1}\left(c_{2}, c_{2}\right)$. Thus, for any state in $P^{*}$, player 1 will not deviate to a state in $P \backslash P^{*}$. Firm 2 makes zero profits for any point in $P^{*}$ and non-positive profits for any $p_{2} \in P_{2}$ if $p_{1} \leq c_{2}$. Thus, Firm 2 has no profitable deviation. This shows deterrence of external deviations for $P^{*}$. 
It remains to verify asymptotic external stability. Let some $\left(p_{1}, p_{2}\right) \in P \backslash P^{*}$ be given. If $c_{2}<p_{1}<p_{2}$, then Firm 2 can profitably deviate to $p_{2}^{\prime}=$ $\left(c_{2}+\min \left\{p_{1}, p_{\mathrm{m}}\right\}\right) / 2$ and Firm 1 can profitably deviate in the next step to $p_{1}^{\prime}=\left(c_{2}+p_{2}^{\prime}\right) / 2$ and so forth. It follows that $\left(c_{2}, c_{2}\right) \in f^{\infty}\left(p_{1}, p_{2}\right) \cap P^{*}$. If $p_{1} \leq c_{2}<p_{2}$, then Firm 1 can profitably deviate to $\left(p_{1}^{\prime}, p_{2}\right)$, where $p_{1}^{\prime} \in\left(c_{2}, p_{2}\right)$ is chosen, such that $p_{1}^{\prime}<c_{2}+\varepsilon$ with $\varepsilon>0$, such that the profits $\left(p_{\mathrm{m}}-c_{1}\right) D\left(p_{\mathrm{m}}\right)$ of a monopolist with marginal costs $c_{1}$ are strictly increasing in $p_{\mathrm{m}}$ on the domain $\left[c_{1}, c_{2}+\varepsilon\right]$. We can then continue as in the previous case. If $c_{2}<p_{2} \leq p_{1}$, then there is $p_{1}^{\prime} \in\left(c_{2}, p_{2}\right)$, such that $\pi_{1}\left(p_{1}^{\prime}, p_{2}\right)>\pi_{1}\left(p_{1}, p_{2}\right)$. Either $p_{2} \leq \bar{p}_{\mathrm{m}}$ or $p_{1}^{\prime}$ is obtained by slightly undercutting $p_{2}$, or $p_{2}>\bar{p}_{m}$ and any $p_{1}^{\prime} \in\left(c_{2}, \bar{p}_{\mathrm{m}}\right)$ will do. Now, we can continue as before. If $p_{1} \notin\left[p_{1}, c_{2}\right]$ and $p_{2}=c_{2}$, then Firm 1 can profitably deviate to $c_{2}$ to reach a strategy profile in $P^{*}$. Because of the legal restrictions on predatory pricing, we have covered all strategy profiles in $P \backslash P^{*}$ and thereby shown that $P^{*}$ satisfies asymptotic external stability.

Step 2. Let $M \subseteq P^{*}$ be a set satisfying deterrence of external deviations and asymptotic external stability. Let us show that, for every $p_{1} \in\left[\underline{p}_{1}, c_{2}\right)$, it holds that $\left(p_{1}, c_{2}\right) \in M$ implies $\left(c_{2}, c_{2}\right) \in M$. Suppose that $\left(p_{1}, c_{2}\right) \in M$ and $\left(c_{2}, c_{2}\right) \notin M$. By closedness of $M$, there is $\varepsilon^{\prime}>0$, such that, for every $\varepsilon \in\left(0, \varepsilon^{\prime}\right),\left(c_{2}-\varepsilon, c_{2}\right) \notin M$. Take $p_{1}^{\prime}=\max \left\{\left(p_{1}+c_{2}\right) / 2, c_{2}-\varepsilon^{\prime} / 2\right\}$, then $\left(p_{1}^{\prime}, c_{2}\right) \in f\left(p_{1}, c_{2}\right)$, and so, $\left(p_{1}^{\prime}, c_{2}\right) \in M$. Given that $p_{1}^{\prime}>c_{2}-\varepsilon^{\prime}$, we obtain a contradiction.

Step 3. Let $M \subseteq P^{*}$ be a set satisfying deterrence of external deviations and asymptotic external stability. Let us show that if $\left(c_{2}, c_{2}\right) \in M$, then, for every $p_{1} \in\left[\underline{p}_{1}, c_{2}\right)$, we have $\left(p_{1}, c_{2}\right) \in M$. This follows from the fact that, for every $p_{1} \in\left(\underline{p}_{1}, c_{2}\right), \pi_{1}\left(p_{1}, c_{2}\right)>\pi_{1}\left(c_{2}, c_{2}\right)$ and the fact that $M$ is closed.

Step 4. We are now ready to show that $P^{*}$ is an MSS. First of all, by Step 1, it satisfies deterrence of external deviations and asymptotic external stability. Therefore, if $P^{*}$ is not an MSS, it should violate minimality. This means that there is a proper subset $M$ of $P^{*}$ that also satisfies deterrence of external deviations and asymptotic external stability. The set $M$ either contains $\left(c_{2}, c_{2}\right)$ or it is a subset of $\left[p_{1}, c_{2}\right) \times\left\{c_{2}\right\}$. If $M$ contains $\left(c_{2}, c_{2}\right)$ then, by Step 3 , it should contain $\left[\underline{p}_{1}, \bar{c}_{2}\right) \times\left\{c_{2}\right\}$ and, therefore, be equal to $P^{*}$, a contradiction with $M$ being a proper subset of $P^{*}$. If $M$ is a subset of $P^{*} \backslash\left\{\left(c_{2}, c_{2}\right)\right\}$, then, by Step 2, it should contain $\left(c_{2}, c_{2}\right)$, a contradiction.

Step 5. Finally, we show that $P^{*}$ is the unique MSS. Let $M$ be an MSS. We show that $P^{*} \cap M \neq \emptyset$. Towards a contradiction, suppose that $P^{*} \cap M=\emptyset$. Then, for all $p^{\prime} \in P^{*}$, there is $p^{\prime \prime} \in M$, such that $p^{\prime \prime} \in f^{\infty}\left(p^{\prime}\right)$. Given that $P^{*}$ is closed and $p^{\prime \prime} \notin P^{*}$, there is $\varepsilon>0$, such that $B_{\varepsilon}\left(p^{\prime \prime}\right) \cap P^{*}=\emptyset$, where $B_{\varepsilon}\left(p^{\prime \prime}\right)=\left\{p \in P \mid d\left(p, p^{\prime \prime}\right)<\varepsilon\right\}$. By definition of $f^{\infty}$, there is $k \in \mathbb{N}$ and $p \in P$, such that $p \in f^{k}\left(p^{\prime}\right)$ and $p \in B_{\varepsilon}\left(p^{\prime \prime}\right)$. By a $k$-fold application of deterrence of external deviations, it holds that $p \in P^{*}$, so $p \in B_{\varepsilon}\left(p^{\prime \prime}\right) \cap P^{*}$ and we have obtained a contradiction. Consequently, it holds that $P^{*} \cap M \neq \emptyset$.

Step 6. If $M$ contains $\left(c_{2}, c_{2}\right)$, then, by Step 3, $M$ should also contain $P^{*} \backslash\left\{\left(c_{2}, c_{2}\right)\right\}$, so $P^{*} \subseteq M$ and by minimality $P^{*}=M$. If $M$ contains an element of $P^{*} \backslash\left\{\left(c_{2}, c_{2}\right)\right\}$, then, by Step 2 , it should also contain $\left(c_{2}, c_{2}\right)$ and, by 
Fig. 1 The MSS for the asymmetric Bertrand model

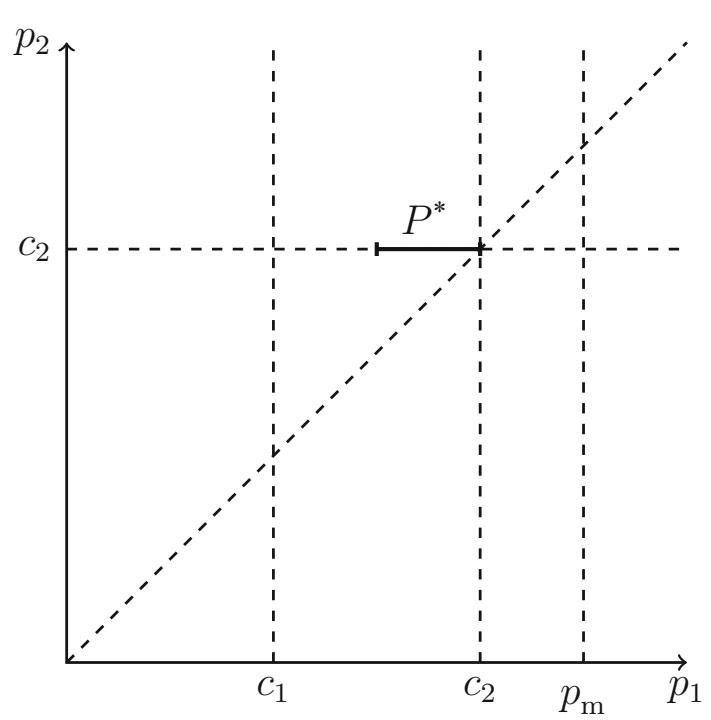

Step 3, also $P^{*} \backslash\left\{\left(c_{2}, c_{2}\right)\right\}$. Again, we obtain $P^{*} \subseteq M$ and by minimality $P^{*}=M$.

For the case $c_{1}=c_{2}=c$, the MSS yields a unique prediction which coincides with the well-known reasoning that prices are equal to marginal cost. This is also the pure strategy Nash equilibrium of the game.

For $c_{1}<c_{2}$, the Myopic Stable Set is illustrated in Fig. 1. The set is relatively small. In any element of the set, firm 2 chooses price equal to its marginal cost. Firm 1 can choose any price between $\underline{p}_{1}$ and $c_{2}$. To provide an intuition, we start with the point $\left(c_{2}, c_{2}\right)$. From this point, $p_{1} \in\left(\underline{p}_{1}, c_{2}\right)$ are all better responses (with $\underline{p}_{1}$ included by closedness). From these points, increasing $p_{1}$ to a value below $c_{2}$ is again all better responses. Finally, $\left(c_{2}, c_{2}\right)$ is included by closedness. The proof shows that, from any point outside the set, there is a sequence of myopic improvements that approaches the set.

In contrast to the unique MSS, the complete set of Nash equilibria has not been characterized. However, as Kartik (2011) shows, there are infinitely many Nash equilibria in mixed strategies which share the property that Firm 1 chooses $p_{1}=c_{2}$ and Firm 2 chooses a mixed strategy with support starting at $c_{2}$. Thus, for the Bertrand game without predatory pricing, the MSS provides intuitive predictions which are less ambiguous than the Nash equilibrium predictions.

In all profiles contained in the MSS, the profit of Firm 1 is strictly lower than its Nash equilibrium profit. Among the profiles in the MSS, the profit of Firm 1 increases as $p_{1}$ approaches $c_{2}$ from below. In the limit, the profit of Firm 1 even approaches its Nash equilibrium profit. 


\section{Discussion}

We have provided a complete characterization of the Myopic Stable Set for the Bertrand duopoly with asymmetric marginal costs. Despite the popularity of Bertrand competition, the set of Nash equilibria has not been fully characterized, but, infinitely, many equilibria exist.

The Myopic Stable Set yields a unique set-valued prediction. The market price is predicted to be weakly lower than in any Nash equilibria in undominated strategies. A clean solution to a game with such a complex set of Nash equilibria boosts the appeal of the Myopic Stable Set as a solution concept.

Open Access This article is distributed under the terms of the Creative Commons Attribution 4.0 International License (http://creativecommons.org/licenses/by/4.0/), which permits unrestricted use, distribution, and reproduction in any medium, provided you give appropriate credit to the original author(s) and the source, provide a link to the Creative Commons license, and indicate if changes were made.

\section{References}

Bertrand, J. (1883). Review of 'Théorie Mathématique de la Richesse Sociale' and 'Recherches sur les Principes Mathématiques de la Théorie des Richesses. Journal des Savants, 67, 499-508.

Blume, A. (2003). Bertrand without fudge. Economics Letters, 78, 167-168.

Chwe, M. S.-Y. (1994). Farsighted coalitional stability. Journal of Economic Theory, 63, 299-325.

Demuynck, T., Herings, P. J. J., Saulle, R. D., \& Seel, C. (2019). The myopic stable set for social environments. Econometrica, 87, 111-138.

Kartik, N. (2011). A note on undominated bertrand equilibria. Economics Letters, 111, 125-126.

Publisher's Note Springer Nature remains neutral with regard to jurisdictional claims in published maps and institutional affiliations. 